


\section{Análisis, modelado y simulación de un sistema de colas en el sector bancario utilizando el software ProModel}

Frank Camilo Atencio Loreth ${ }^{1}$, Daniel Eduardo Bolaño Villalba ${ }^{2}$, Gean Pablo Mendoza Ortega ${ }^{3}$, Eduard de Jesús Díaz Ramos ${ }^{4}$

\section{Resumen}

Esta investigación se centró en analizar y simular el comportamiento de una línea de espera de un banco, utilizando softwares estadísticos y de simulación como ProModel y StatFit. Los resultados hallados sugieren una modificación del modelo actual (M/M/3) a uno que provea más servidores para distribuir mejor el exceso de carga en los servidores. De esta investigación se concluye que la configuración del sistema debe tener un mínimo de 18 servidores, M/M/18, para tener una mejora sustancial de la calidad del servicio, reduciendo los tiempos de espera de los clientes a un 59.73\% en comparación con el 88.8\% del modelo actual (M/M/3).

Palabras clave: modelamiento de sistemas, ProModel, simulación por computadora, teoría de colas.

$1 \quad$ Ingeniero Industrial. Corporación Universitaria del Caribe-CECAR. Correo: Frank.atencio@cecar.edu.co; Orcid: https://orcid.org/0000-0002-9274-5215

2 Ingeniero Industrial. Corporación Universitaria del Caribe-CECAR. Correo: daniel.bolanov@cecar.edu.co; Orcid: https://orcid.org/0000-0002-8621-0388

3 Estudiante PhD. en Logística y gestión de cadenas de suministro, Maestría en logística integral. Ingeniero Agroindustrial. Universidad de Sucre. Docente investigador de la Corporación Universitaria del Caribe - CECAR. Facultad Ciencias Básicas, Ingenierías y Arquitectura. Grupo de Investigación Gestión de la Innovación Empresarial y Social-GIES. Categorizado por Minciencias en el nivel Junior. Correo: Gean.mendoza@ cecar.edu.co; Orcid: https://orcid.org/0000-0002-4601-6185

$4 \quad$ Magíster en Logística Integral. Ingeniero Industrial. Corporación Universitaria del Caribe-CECAR. Correo: eduard.diaz@cecar.edu.co; Orcid: https://orcid.org/00000001-9683-578X 


\section{Analysis, modeling and simulation of a queuing system in the banking sector using ProModel software}

Abstract

This research focused on analyzing and simulating the behavior of a bank waiting line, using statistical and simulation software such as ProModel and StatFit. The results found suggest a modification of the current model $(\mathrm{M} / \mathrm{M} / 3)$ to one that provides more servers to better distribute the excess load on the servers. From this research it is concluded that the system configuration must have a minimum of 18 servers, $M / M / 18$, to have a substantial improvement in the quality of service, reducing the waiting times of the clients to $59.73 \%$ compared to $88.8 \%$ of the current model (M/M/3).

Keywords: computer simulation, ProModel, systems modeling, queuing theory. 


\section{Introducción}

En la vida cotidiana se presenta un fenómeno muy ocurrente: las líneas de espera. Esto se origina cuando la demanda del servicio supera la disposición existente de quienes prestan un servicio, por ejemplo, los tiempos de espera en un semáforo y las colas de atención al cliente en establecimientos comerciales, bancarios o de cualquier otra índole. Las áreas más destacadas que emplean la teoría de colas son los hospitales y las entidades bancarias debido a la alta ocurrencia de eventos discretos y no discretos, y a que los servidores deben actuar lo más rápido posible, así como tomar decisiones con la misma prontitud con la que actúan (Gómez, 2011; Jahn, Theurl, Siebert \& Pfeiffer 2010).

Este proceso de generación de líneas de espera trae consigo diferentes tipos de inconvenientes que se reflejan a corto y mediano plazo. Por tal motivo, se cuenta con un conjunto de modelos matemáticos que se enmarcan en el área de "la teoría de colas" (De Arias, Montoya, \& Hen, 2010).

El tener que esperar en una cola es una experiencia que se considera desagradable, especialmente si se tiene que esperar de pie. Los periodos largos de espera irritan a las personas y las invitan a desertar, a irse a otra parte e incluso a no regresar. Esta situación que afecta los beneficios potenciales puede gestionarse en forma eficiente utilizando la teoría de colas (Orrego, 2012)

El objetivo de la teoría de colas es modelar sistemas de espera que funcionen de la siguiente manera: existe un medio al que llegan clientes demandando cierto servicio. Luego, a consecuencia de que la demanda no puede ser satisfecha inmediatamente, se forma una cola (o línea de espera) de clientes en espera de ser atendidos por el o los servidores correspondientes. Los tiempos entre arribo de clientes consecutivos al sistema y los tiempos de servicio son aleatorios y son representados por variables aleatorias con alguna distribución de probabilidad (Abad, 2002).

Durante la segunda etapa de la revolución industrial (1870 1914), el crecimiento exponencial de la industria comenzó a ser notable y era necesario tener en cuenta el control de la calidad del producto y del proceso mismo. En ese entonces se desarrollaron muchos métodos a partir 
de la ingeniería metódica, entre ellos, la teoría de colas que se utilizó para obtener información acerca de los cuellos de botella presentes en las líneas de montajes, para posteriormente optimizar dichas líneas, logrando agilizar el ensamblaje de vehículos (Cerutti \& Valdaliso, 2003).

El origen de la teoría de colas se remonta a un estudio realizado por el matemático danés Agner Krarup Erlang (Dinamarca, 1878 - 1929), en el cual se estudiaba el tráfico de llamadas telefónicas con el propósito de apaciguar la demanda del servicio en la ciudad de Copenhague. El resultado de este estudio fue el nacimiento de esta teoría la cual corresponde a un tópico de la investigación operativa y se utiliza actualmente como herramienta de optimización de tiempos en las líneas de espera (Benites \& Virhuez, 2019)

La simulación por computadora ha sido empleada muchas veces en distintas áreas, enfocándose en sistemas de manufactura y servicios. El uso de la computación para la simulación de sistemas ha tenido un gran desarrollo durante los últimos años debido al crecimiento progresivo de las herramientas de cómputo, además, esta tiene la capacidad de incluir información adicional que no se ajusta a modelos tradicionales de la teoría de colas. Los modelos tradicionales son aquellos cuyo comportamiento conlleva a tener resultados erróneos en el análisis de las medidas de desempeño (Pérez \& Riaño, 2007; Márquez, 2010).

La importancia de esta teoría radica en la capacidad de modelar un sistema, abstraerlo en términos matemáticos y simularlo en un software de simulación como ProModel; dicho modelo, acompañado de datos recolectados del sistema real, nos permite obtener una base teórica y simulada para evaluar y comparar con el modelo real, encontrando similitudes o discrepancias que arrojen una respuesta para la toma de decisiones. Dicha simulación sirve para no comprometer el sistema real y mejorarlo o corregirlo con base a los resultados obtenidos del modelo simulado.

Para todas las empresas que prestan un servicio, el número de clientes complacidos o satisfechos es un factor sustancial al momento de ofrecer productos o servicios, maximizándolos cuando se trata de productos inmateriales, en los que el ambiente que rodea la entrega del producto sea lo que permita generar el valor agregado. En tales circunstancias, dicho valor 
agregado se fundamentará en controlar el tiempo de espera de atención a un cliente o usuario.

Dada la alta demanda de usuarios y a la capacidad de los servidores con los que cuenta Bancamía, una sucursal bancaria localizada en la ciudad de Sincelejo, se presentan demoras en el servicio de atención al cliente. Esta situación aqueja y deja insatisfechos a muchos de sus usuarios, resultando en la notable afectación de la imagen de la entidad bancaria y ocasionando una posible pérdida de usuarios. Para la realización de sus actividades la sucursal cuenta con un total de 3 servidores, quienes se encargan de atender a los usuarios. Además, la sucursal planea introducir un servidor extra para dar una solución al problema en búsqueda de mejorar el servicio. A partir de una indagación, se conoció que la sucursal no cuenta con estudios anteriores que den una solución a su problema, por ello, se les presentó una propuesta de estudio basada en el análisis y simulación de sus tiempos de atención al cliente con el fin de obtener resultados y realizar recomendaciones para la mejora del servicio.

En muchas ocasiones, lo que determina el éxito o el fracaso de una empresa es la calidad de sus servicios. La razón de este estudio aborda la calidad del servicio al cliente de la sucursal bancaria Bancamía, con el propósito de identificar los problemas que producen los cuellos de botella en el sistema. Los cuellos de botella son aquellos elementos que perjudican un proceso dentro de una empresa, ocasionando que se disminuya la producción y se incrementen los tiempos de espera, lo cual reduce la productividad, generando costos extras (Gavilanes, Acosta, Gaibor, \& Tenorio, 2018). Al identificar estos cuellos de botella, la entidad evitará contratiempos y reducirá sus pérdidas. Además, con la simulación del sistema se evita comprometer el sistema real.

El objetivo principal de esta investigación consiste en analizar y simular la conducta de una línea de espera. También se pretende tomar muestras de los tiempos de espera de los clientes para luego modelar un sistema utilizando software de simulación y software estadístico, obteniendo así resultados que nos permitan realizar una propuesta de mejora en el servicio e identificar si es necesaria la implementación de más servidores en la sucursal. 
La estructura de este documento se distribuye así: al inicio se presenta una sección introductoria; seguida de una sección donde se describe la metodología empleada; un apartado referente a los resultados; después, la conclusión y las recomendaciones; y, por último, la bibliografía.

\section{Materiales o metodología}

La metodología empleada se basó en la metodología de planeación del modelo de simulación propuesto por García Dunna y Azarang Esfandiari (1996), la cual está compuesta por una descripción del sistema, la formulación del modelo, la recolección de datos, la codificación del modelo y, por último, la obtención de resultados y experimentación. A continuación, se describen las etapas mencionadas anteriormente.

\section{Descripción del sistema}

En cuanto a la descripción del sistema, se procedió a identificar los elementos, considerando que el sistema cuenta con tres servidores y una sola línea de espera, teniendo un sistema de colas tipo M/M/3, en la que un conjunto de $\mathrm{N}$ clientes ingresan al sistema en busca de un servicio, estos esperan en una cola hasta que sea su turno y abandonan el sistema una vez son atendidos.

\section{Formulación del modelo}

Por otra parte, para la formulación del sistema se procedió con la identificación de las locaciones, las entidades, los procesos, los recursos, los arribos y la forma en que estos se distribuyen a lo largo del sistema. A continuación, se muestra, a manera de diagrama de flujo, el sistema en general, seguido de una forma más detallada, (figura 1 y figura 2). 
Figura 1

Diagrama de flujo del sistema.

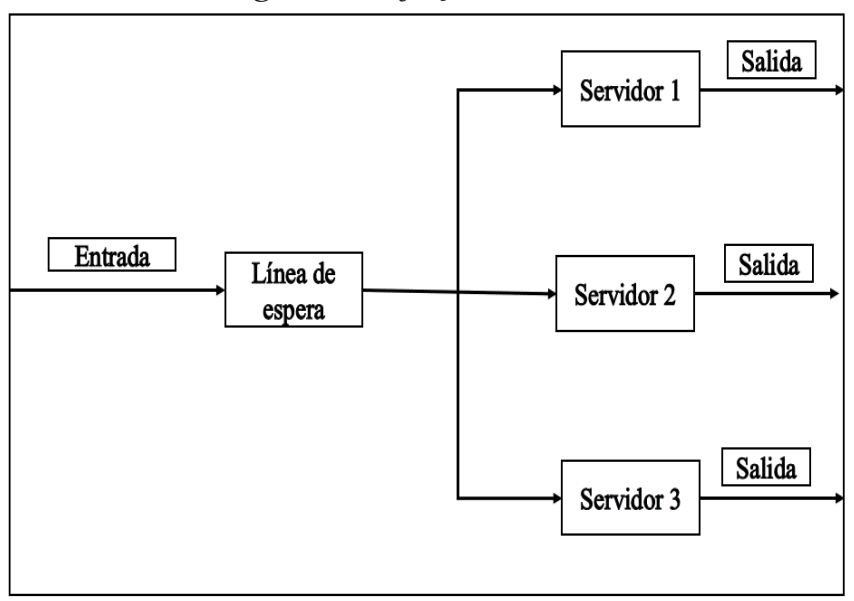

Nota. Fuente: Atencio, Bolaño y Díaz.

Figura 2

Diagrama de flujo detallado del sistema.

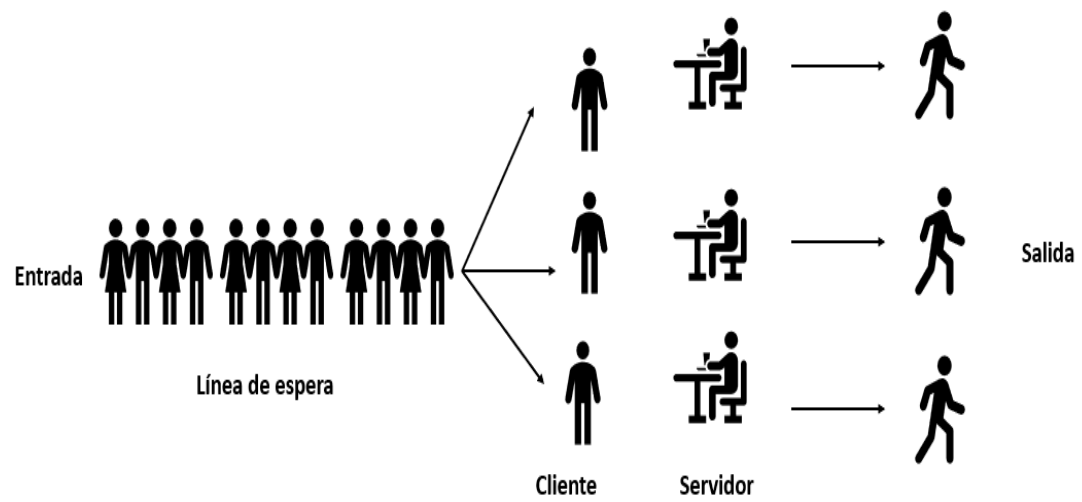

Nota. Fuente: Atencio, Bolaño y Díaz.

\section{Recolección de datos}

Referente a la recolección de los datos, se tomaron tres mediciones de tiempo utilizando un cronometro, dados en minutos: el tiempo entre llegadas al sistema, el tiempo de espera en cola y el tiempo de atención. Se registró una muestra de cuarenta muestras durante una semana laboral (lunes - viernes), obedeciendo al horario laboral (8:00 a.m. - 4:00 p.m.) Los datos fueron tabulados en Microsoft Excel y luego se realizó un análisis de 
ajuste para encontrar las distribuciones con el software estadístico EasyFit. Las mediciones y distribuciones de probabilidad se pueden observar detalladamente en la sección de resultados.

\section{Codificación del modelo en PC}

En las siguientes figuras se mostrará la codificación del modelo planteado en la formulación del modelo haciendo uso del software de simulación ProModel.

\section{Locaciones}

En esta sección se definieron y configuraron dos locaciones: la primera, la línea de espera; y, la segunda, los servidores.

Figura 3

Definición de locaciones utilizando ProModel.

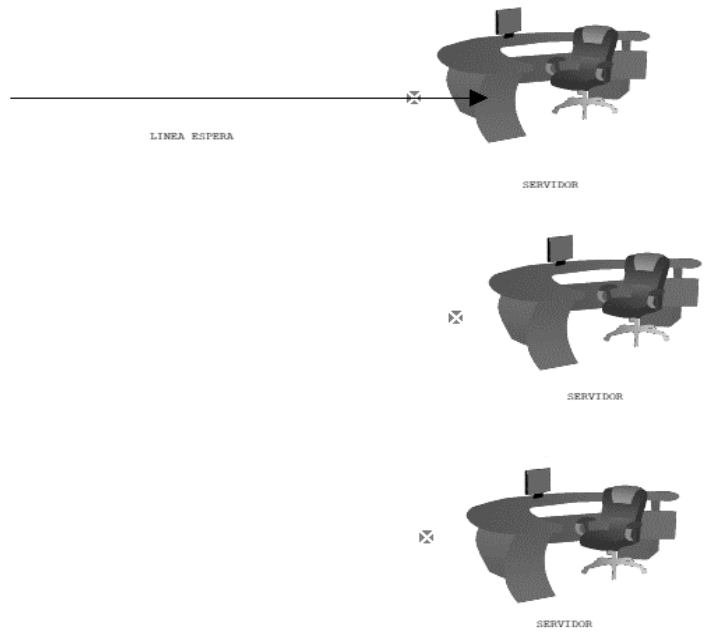

Nota. Fuente: Atencio, Bolaño y Díaz. 
Análisis, modelado y simulación de un sistema de colas en el sector bancario utilizando el software ProModel

\section{Figura 4}

Definición de locaciones utilizando ProModel.

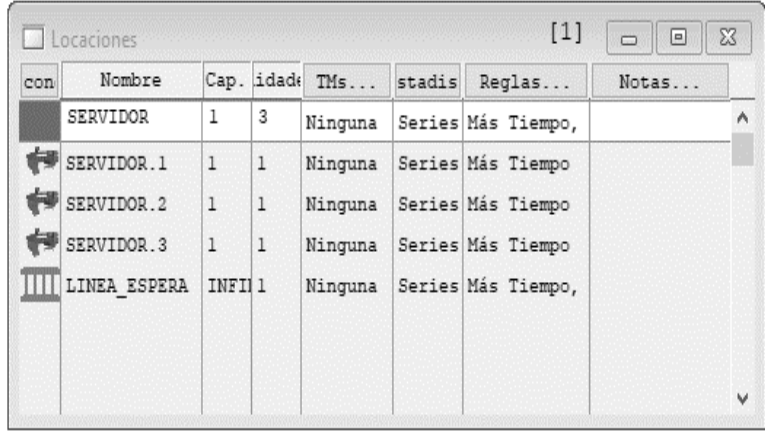

Nota. Fuente: Atencio, Bolaño y Díaz.

\section{Entidades}

En esta sección se definió la entidad, quien es el cliente que recibe el servicio.

\section{Figura 5}

Definición de entidades utilizando ProModel.

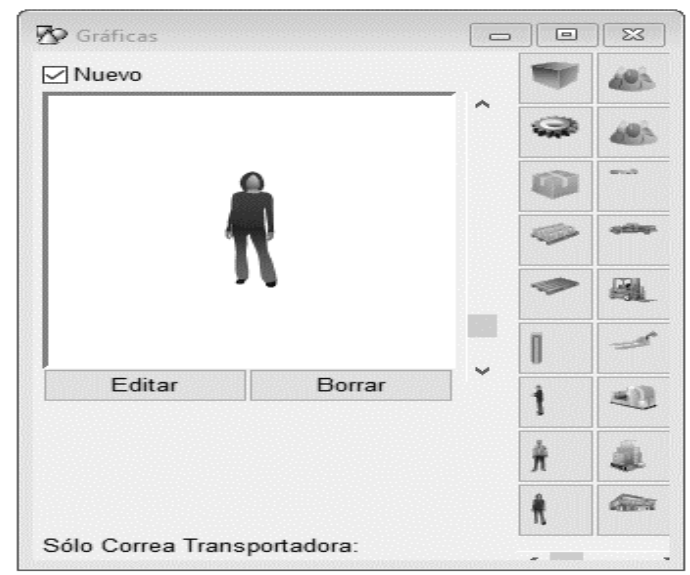

Nota. Fuente: Atencio, Bolaño y Díaz. 
Figura 6

Configuración de entidades utilizando ProModel.

\begin{tabular}{|c|c|c|c|c|c|c|}
\hline \multicolumn{3}{|c|}{ Entidades } & [1] & 口 & 回 & 83 \\
\hline or & Nombre & ocidad ( & Estadist & & ;as. . & \\
\hline & IENTE & 150 & Series de & & & $\wedge$ \\
\hline & & & & & & $v$ \\
\hline
\end{tabular}

Nota. Fuente: Atencio, Bolaño y Díaz.

\section{Llegadas o arribos}

En esta sección se configura el comportamiento de cómo los clientes llegan al sistema.

\section{Figura 7}

Definición de arribos utilizando ProModel.

\begin{tabular}{|l|l|}
\hline So Herramientas \\
Entidad: \\
\hline CLIENTE \\
\hline ALL \\
\hline CLIENTE \\
\hline
\end{tabular}

Nota. Fuente: Atencio, Bolaño y Díaz.

\section{Figura 8}

Configuración de arribos utilizando ProModel.

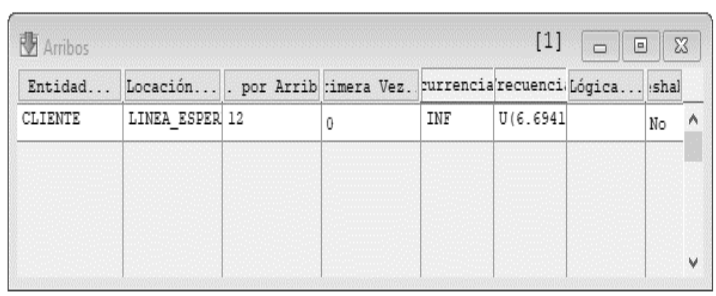

Nota. Fuente: Atencio, Bolaño y Díaz.

\section{Procesos}

En esta sección se definen los procesos del modelo. Estos incluyen la llegada, la espera y la atención del cliente. 
Análisis, modelado y simulación de un sistema de colas en el sector bancario utilizando el software ProModel

\section{Figura 9}

Configuración de proceso utilizando ProModel. Pt.1

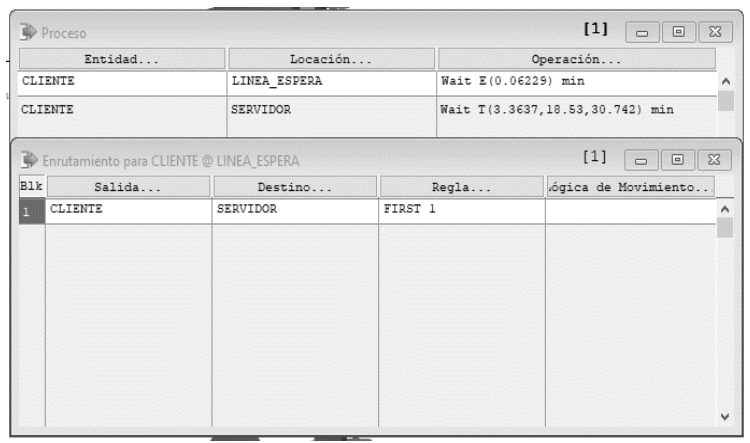

Nota .Fuente: Atencio, Bolaño y Díaz.

Figura 10

Configuración de proceso utilizando ProModel. Pt.2

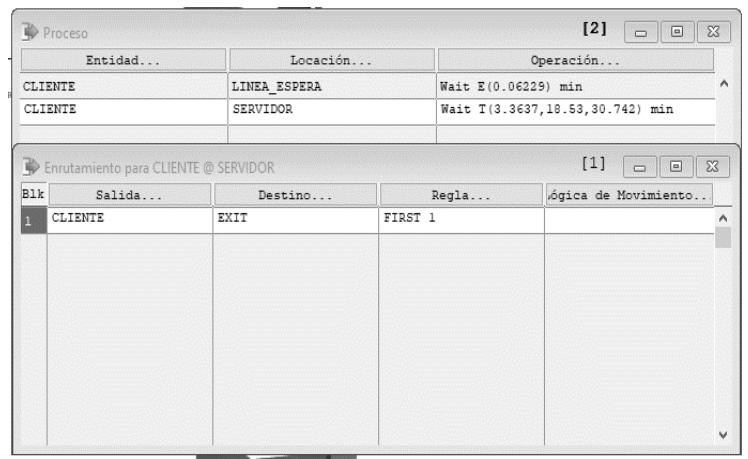

Nota. Fuente: Atencio, Bolaño y Díaz

\section{Resultados y análisis}

En este apartado se muestran los resultados obtenidos de las mediciones y del modelo descrito anteriormente.

\section{Mediciones}

Las mediciones de los tiempos están dadas en minutos y están tabuladas en las siguientes tablas; luego, se presentan una gráfica que describe el comportamiento de los datos tabulados y una tabla de distribución de 
probabilidades según el tipo de medición, producto de un análisis con EasyFit para ajustar los datos a una distribución de probabilidad.

Tabla 1

Mediciones de los tiempos entre llegadas.

\begin{tabular}{|c|c|c|c|c|}
\hline Día 1 & Día 2 & Día 3 & Día 4 & Día 5 \\
\hline 21 & 24,75 & 11,23 & 5,95 & 18,95 \\
\hline 9,44 & 16,4 & 4,54 & 48,01 & 22,08 \\
\hline 11,66 & 27,01 & 14,77 & 27,22 & 67,45 \\
\hline 8,43 & 19,7 & 3,46 & 8,89 & 50,23 \\
\hline 9,86 & 10,07 & 33,95 & 11,3 & 39,9 \\
\hline 13,97 & 18,02 & 36,76 & 33,81 & 47,01 \\
\hline 10,3 & 3,51 & 12,76 & 27,46 & 67,7 \\
\hline 5,48 & 11,33 & 25,14 & 7,98 & 63,84 \\
\hline 10,96 & 25,06 & 6,25 & 28,2 & 13,49 \\
\hline 10,15 & 23,5 & 39,71 & 10,09 & 18,27 \\
\hline 25,14 & 11,73 & 38,4 & 39,75 & 26,36 \\
\hline 10,46 & 10,23 & 17,17 & 45,91 & 45,45 \\
\hline 5,69 & 23,89 & 25,89 & 15,78 & 23,8 \\
\hline 24,89 & 6,56 & 6,49 & 48,47 & 25,78 \\
\hline 23,74 & 15,56 & 38,14 & 21,79 & 60,51 \\
\hline 28,42 & 11,53 & 33,4 & 7,83 & 29,92 \\
\hline 9,33 & 28,15 & 14,71 & 44,15 & 16,29 \\
\hline 8,36 & 18,39 & 17,52 & 22,32 & 43,08 \\
\hline 24,31 & 28,11 & 23,33 & 12,17 & 21,77 \\
\hline 25,3 & 3,45 & 8,1 & 24,91 & 19,46 \\
\hline 23,6 & 6,34 & 26,19 & 17,65 & 40,67 \\
\hline 18,01 & 29,74 & 3,44 & 23,76 & 3,39 \\
\hline 20,11 & 22,9 & 29,99 & 24,79 & 62,07 \\
\hline 26,88 & 28,32 & 37,16 & 35,33 & 36,87 \\
\hline 9,14 & 24,63 & 25,98 & 2,05 & 64,97 \\
\hline 18,06 & 9,03 & 36,99 & 45,42 & 17,51 \\
\hline 16,91 & 10,05 & 39,64 & 27,1 & 29,75 \\
\hline 7,93 & 13,82 & 38,82 & 23,81 & 26,06 \\
\hline 7,99 & 19,96 & 16,75 & 17,53 & 52,59 \\
\hline
\end{tabular}


Análisis, modelado y simulación de un sistema de colas en el sector bancario utilizando el software ProModel

\begin{tabular}{ccccc}
\hline Día 1 & Día 2 & Día 3 & Día 4 & Día 5 \\
\hline 28,62 & 22,6 & 6,97 & 35,13 & 26,88 \\
26,08 & 15,15 & 29,26 & 8,82 & 50,55 \\
17,95 & 3,79 & 3,19 & 5,4 & 34,45 \\
8,79 & 15,34 & 31,07 & 7,24 & 2,07 \\
20,21 & 24,97 & 27,31 & 41,87 & 45,95 \\
16,4 & 21,78 & 15,98 & 2,1 & 48,94 \\
16,56 & 28,79 & 24,5 & 38,32 & 36,61 \\
23,59 & 27,81 & 19,71 & 32,62 & 42,72 \\
25,01 & 11,04 & 9,49 & 22,15 & 66,39 \\
6,84 & 3,61 & 8,27 & 21,87 & 24,99 \\
6,63 & 8,02 & 22,66 & 10,27 & 59,09 \\
\hline
\end{tabular}

Nota. Fuente: Atencio, Bolaño y Díaz.

Tabla 2

Mediciones de los tiempos en línea de espera.

\begin{tabular}{ccccc}
\hline Día 1 & Día 2 & Día 3 & Día 4 & Día 5 \\
\hline 14,42 & 27,48 & 24,55 & 45,49 & 4,48 \\
10,13 & 22,03 & 27,94 & 20,14 & 6,82 \\
9,81 & 2,96 & 8,64 & 9,37 & 54,42 \\
6,67 & 4,81 & 18,52 & 15,22 & 19,69 \\
17,75 & 5,91 & 39,39 & 24,31 & 25,62 \\
16,51 & 9,7 & 10,16 & 9,08 & 28,61 \\
10,85 & 12,67 & 15,03 & 42,86 & 64,13 \\
10,1 & 10,29 & 21,72 & 18,48 & 37,52 \\
23,88 & 30,29 & 17,67 & 9,92 & 17,91 \\
21,04 & 4,05 & 1,87 & 2,99 & 32,78 \\
17,21 & 22,16 & 14,62 & 17,12 & 68,35 \\
27,99 & 22,12 & 8 & 5,44 & 57,89 \\
21,98 & 7,66 & 25,25 & 11,11 & 1,87 \\
13,05 & 18,39 & 39,91 & 47,55 & 13,45 \\
22,36 & 10,06 & 22,06 & 21,11 & 6,03 \\
23,83 & 11,75 & 36,34 & 16,42 & 65,39 \\
28,13 & 10,7 & 34,67 & 4,9 & 50,19
\end{tabular}




\begin{tabular}{ccccc}
\hline Día 1 & Día 2 & Día 3 & Día 4 & Día 5 \\
\hline 18,32 & 28,57 & 23,42 & 11,38 & 18,63 \\
21,23 & 7,57 & 8,65 & 35,42 & 8,47 \\
27,93 & 28,4 & 22,53 & 38,99 & 67,12 \\
9,24 & 7,75 & 35,63 & 19,89 & 41,15 \\
21,71 & 28,06 & 38,48 & 5,43 & 9,01 \\
27,51 & 28,1 & 26,66 & 8,07 & 7,67 \\
15,54 & 8,22 & 32,83 & 35,68 & 21,13 \\
18,42 & 10,23 & 40,11 & 10,07 & 6,74 \\
19,9 & 24,51 & 9,59 & 6,27 & 41,26 \\
28,05 & 30,4 & 33,91 & 6,82 & 33,26 \\
10,3 & 14,86 & 30,42 & 33,79 & 31,24 \\
7,49 & 16,16 & 5,44 & 26,74 & 34,7 \\
17,96 & 16,23 & 15,34 & 17,08 & 11,65 \\
24,19 & 29,76 & 3,8 & 48,66 & 46,33 \\
14,65 & 19,35 & 3,65 & 4,42 & 5,25 \\
22,28 & 5,02 & 11,42 & 11,45 & 39,53 \\
23,83 & 9,19 & 9,09 & 9,16 & 64,48 \\
24,54 & 7,9 & 4,18 & 5,87 & 20,63 \\
5,43 & 17,05 & 30,68 & 8,18 & 5,95 \\
10,91 & 10,9 & 5,47 & 9,03 & 66,61 \\
19,71 & 25,8 & 4,85 & 35,97 & 27,64 \\
26,38 & 3,79 & 11,12 & 15,03 & 34,53 \\
16,95 & 3,4 & 32,28 & 41,53 & 4,77 \\
\hline
\end{tabular}

Nota. Fuente: Atencio, Bolaño y Díaz. 
Análisis, modelado y simulación de un sistema de colas en el sector bancario utilizando el software ProModel

Tabla 3

Mediciones de los tiempos de atención.

\begin{tabular}{ccccc}
\hline Día 1 & Día 2 & Día 3 & Día 4 & Día 5 \\
\hline 16,68 & 25,17 & 37,84 & 44,12 & 43,52 \\
13,16 & 26,41 & 20,7 & 3,37 & 63,06 \\
11,17 & 27,86 & 35,86 & 15,42 & 48,77 \\
20,81 & 23,48 & 40,47 & 22,26 & 40,02 \\
28,39 & 9,71 & 34,65 & 10,15 & 21,42 \\
16,37 & 6,72 & 14,78 & 21,82 & 35,4 \\
6,05 & 26,14 & 32,02 & 33,9 & 57,07 \\
15,95 & 27,23 & 20,04 & 32,22 & 42,81 \\
6,73 & 6,15 & 28,93 & 43,54 & 7,09 \\
25,81 & 21,77 & 27,66 & 32,13 & 7,6 \\
9,38 & 22,49 & 23,39 & 14,1 & 16,34 \\
20,64 & 8,01 & 24,33 & 12,3 & 33,5 \\
23,23 & 18,79 & 21,71 & 10,6 & 27,57 \\
25,92 & 18,21 & 5,44 & 45,32 & 19,74 \\
18,53 & 24,33 & 26,23 & 20,11 & 12,92 \\
21,73 & 19,04 & 9,32 & 6,81 & 2,29 \\
8,36 & 23,5 & 27,2 & 37,37 & 48,3 \\
20,22 & 29,24 & 7,48 & 33,35 & 7,1 \\
18,38 & 3,73 & 35,23 & 29,15 & 13,5 \\
6,1 & 20,71 & 7,29 & 33,16 & 68,67 \\
17,87 & 21,05 & 9,38 & 36,22 & 13,05 \\
25,18 & 16,63 & 19,5 & 1,37 & 6,38 \\
24,4 & 16,16 & 29,35 & 30,79 & 18,12 \\
24,32 & 9,29 & 26,6 & 4,22 & 57,82 \\
18,12 & 19,64 & 17,82 & 35,41 & 20,64 \\
28,28 & 29,32 & 15,35 & 2,78 & 31,51 \\
12,57 & 25,4 & 27,45 & 32,4 & 9,97 \\
22,51 & 12,03 & 15,28 & 27,26 & 61,73 \\
17,4 & 14,54 & 1,75 & 4,26 & 35,36 \\
12,39 & 26,03 & 24,67 & 40,58 & 25,43 \\
20,51 & 6,5 & 11,54 & 16,13 & 22,32
\end{tabular}


Frank Camilo Atencio Loreth Daniel Eduardo Bolaño Villalba

Gean Pablo Mendoza Ortega. Eduard de Jesús Díaz Ramos

\begin{tabular}{ccccc}
\hline Día 1 & Día 2 & Día 3 & Día 4 & Día 5 \\
\hline 16,45 & 3,47 & 26,78 & 9,13 & 29,14 \\
18,54 & 15,51 & 21,67 & 33,19 & 49,3 \\
11,02 & 11,78 & 22,44 & 21,63 & 48,96 \\
16,36 & 7,2 & 14,74 & 37,81 & 63,97 \\
17,21 & 25,52 & 13,22 & 45,84 & 9,07 \\
13,32 & 19,55 & 1,45 & 23,17 & 63,51 \\
13,54 & 16,65 & 22,02 & 49,1 & 63 \\
24,43 & 8,45 & 2,24 & 21,19 & 67,89 \\
22,41 & 11,79 & 6,84 & 27,92 & 69,91 \\
\hline
\end{tabular}

Nota. Fuente: Atencio, Bolaño y Díaz.

Figura 11

Comportamiento de los datos recolectados en los tiempos de llegada, línea de espera y atención.

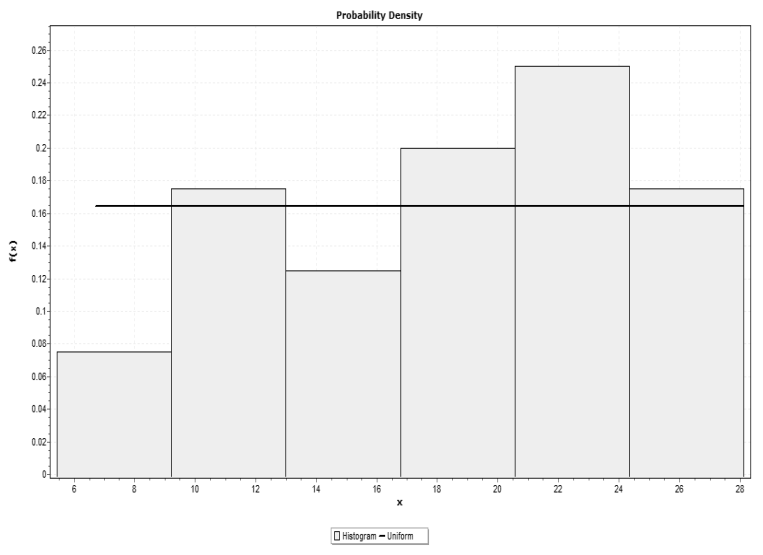



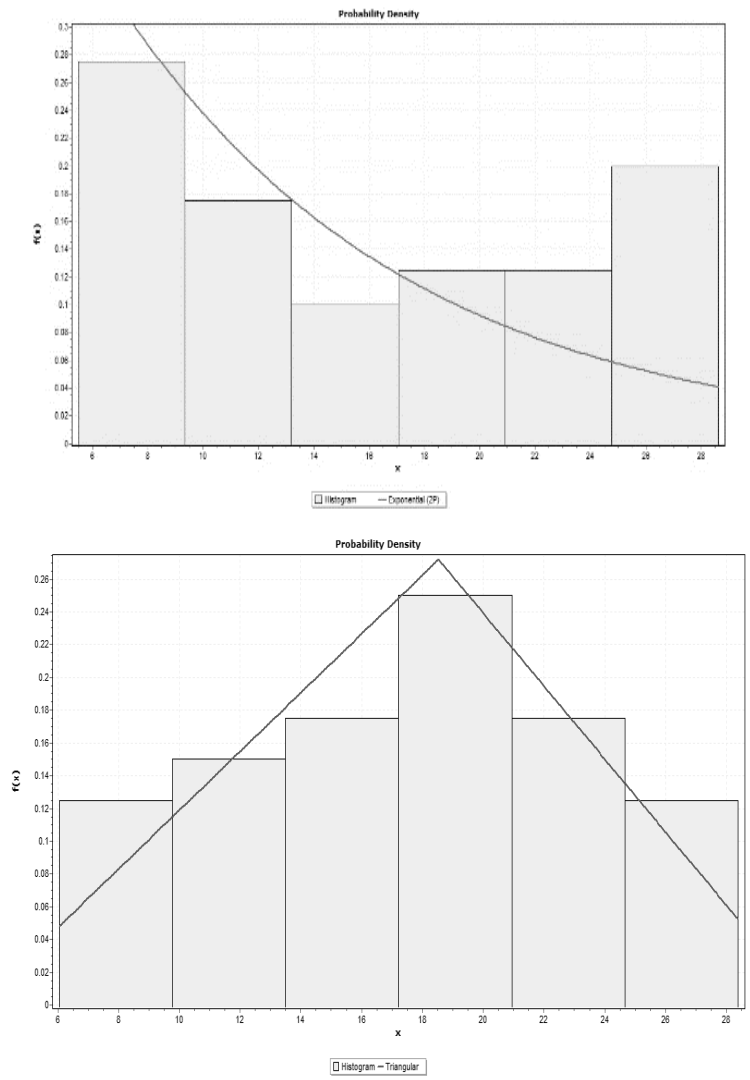

Nota. Fuente: Atencio, Bolaño y Díaz.

\section{Distribuciones de probabilidades}

En la siguiente tabla (tabla 4) se verán reflejadas las distribuciones de probabilidad, junto a los parámetros a emplear en el modelo simulado.

Tabla 4

Distribuciones de probabilidad y parámetros para cada tipo de tiempo.

\begin{tabular}{ccc}
\hline Tipo de tiempo & Distribución & Parámetros \\
\hline $\begin{array}{c}\text { Tiempo entre } \\
\text { llegadas }\end{array}$ & Uniforme & $\mathrm{a}=6.6941, \mathrm{~b}=29.715$ \\
$\begin{array}{c}\text { Tiempo de espera } \\
\text { Tiempo de } \\
\text { atención }\end{array}$ & Exponencial & $\lambda=0.06229$ \\
\hline
\end{tabular}

Nota. Fuente: Atencio, Bolaño y Díaz. 


\section{Validación del modelo de simulación}

En esta sección se realizó una simulación previa de 30 réplicas de 8 horas para obtener información del comportamiento del modelo y compararlo con los datos reales, haciendo uso del teorema de Tchebycheff para el cálculo del número de réplicas y las pruebas de hipótesis, con el fin de corroborar si el sistema se comporta como el sistema real. La variable de observación escogida para esta prueba es el tiempo de atención.

\section{Cálculo del número óptimo de corridas}

La siguiente tabla muestra los parámetros utilizados para el cálculo óptimo de corridas.

\section{Tabla 5}

Parámetros y datos utilizados para el cálculo del número óptimo de corridas.

\begin{tabular}{cc}
\hline Distr. Normal & No \\
\hline Valor de significancia $(\alpha)$ & 0,05 \\
Desviación estándar $(\sigma)$ & 0,748 \\
Error $(\mathrm{E})$ & 0,2 \\
Número de corridas & $279,84 \approx 280$ \\
\hline
\end{tabular}

Nota. Fuente: Atencio, Bolaño y Díaz.

El número de corridas óptimo para la simulación es de aproximadamente 280 .

\section{Prueba de hipótesis}

En este apartado se realizará una prueba de hipótesis de dos colas, basados en la media del sistema real.

$$
\begin{aligned}
& \mathrm{H}_{0}: \mu(\text { modelo })=\mu(\text { real }) \\
& \mathrm{H}_{1}: \mu(\text { modelo }) \neq \mu(\text { real })
\end{aligned}
$$

La siguiente tabla muestra los resultados de la prueba $\mathrm{Z}$ para media de dos colas. 
Tabla 5

Resultados de la prueba $Z$ para media de dos colas.

\begin{tabular}{cc}
\hline $\mathrm{z}$ & 0,057231 \\
\hline $\mathrm{P}(\mathrm{Z} \leq \mathrm{z})$ una cola & 0,477181 \\
Valor crítico de z (una cola) & 1,644854 \\
Valor crítico de z (dos colas) & 0,954361 \\
Valor crítico de z (dos colas) & 1,959964 \\
\hline
\end{tabular}

Nota. Fuente: Atencio, Bolaño y Díaz.

Como se puede observar en la tabla anterior, el Z calculado es menor que el valor crítico, por lo tanto, este valor se encuentra dentro del rango. En consecuencia, no se tienen las suficientes pruebas estadísticas para rechazar la hipótesis nula lo que significa que el sistema simulado se comporta como el sistema real.

\section{Análisis y experimentación de los resultados de la simulación.}

En las siguientes gráficas se muestran los resultados de la simulación utilizando tres servidores y una cola, las cuales se analizarán para posteriormente determinar la viabilidad de incorporar un nuevo servidor en la sucursal.

La siguiente gráfica evidencia que, en promedio, el $88.80 \%$ de los clientes debe esperar, lo cual indica un alto grado de tiempo de espera.

Figura 12

Porcentaje promedio de espera de los clientes en el sistema.

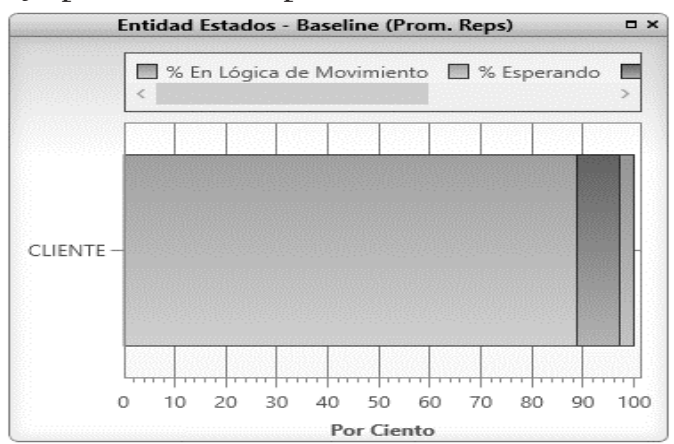

Nota. Fuente: Atencio, Bolaño y Díaz. 
La siguiente gráfica muestra el porcentaje de ocupación de los servidores, teniendo un porcentaje de ocupación cercano al 100\%, con un porcentaje exacto del $99.94 \%$ de ocupación.

\section{Figura 13}

Porcentaje promedio de ocupación de los servidores.

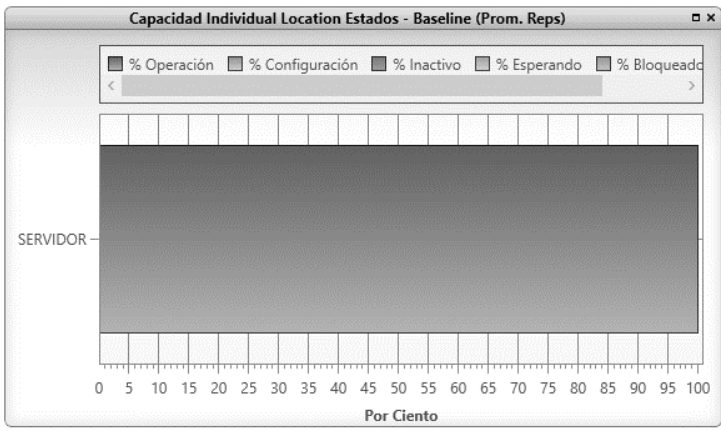

Nota. Fuente: Atencio, Bolaño y Díaz.

La siguiente gráfica muestra la línea de espera con un porcentaje de ocupación del 100\%.

\section{Figura 14}

Porcentaje promedio de ocupación de la línea de espera.

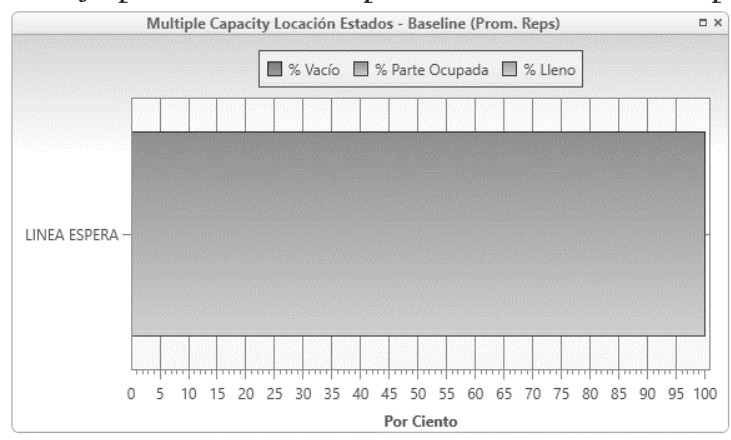

Nota. Fuente: Atencio, Bolaño y Díaz.

Lo anteriormente mostrado en las gráficas 4, 5 y 6 evidencia que el sistema está trabajando casi a su máxima capacidad, con el principal cuello de botella en la atención al cliente. No hay suficientes servidores para atender a todos los usuarios, lo cual hace que el sistema sea lento; es un cuello de botella generado por falta de más servidores. 
A continuación, se muestra una experimentación con el sistema, de tal forma que se aumentara el número de servidores para contrastar con el comportamiento del sistema de 3 servidores (M/M/3). En esta experimentación se busca reducir el tiempo de espera del cliente y el tiempo de atención distribuyendo la carga en más servidores.

Adicionando 12 servidores al sistema, se tiene que los porcentajes de ocupación de los servidores y de la línea de espera disminuyen con respecto al modelo actual a la vez que el porcentaje de espera por parte del cliente se aminora. Se cambió el modelo M/M/1 a M/M/15 para la experimentación. A continuación, se muestran las gráficas obtenidas de la simulación del modelo M/M/15.

Para el cliente se tiene un $70.12 \%$, un $18.68 \%$ menos que en el modelo principal de tres servidores y una cola.

\section{Figura 15}

Porcentaje promedio de espera de los clientes en el sistema, modelo de 12 servidores y 1 cola.

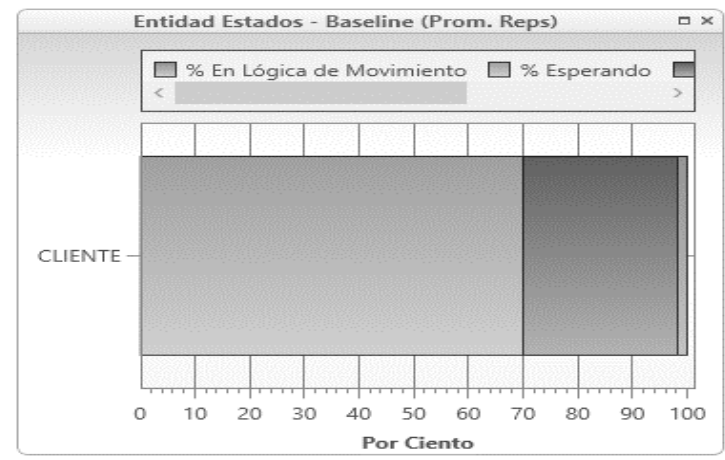

Nota. Fuente: Atencio, Bolaño y Díaz.

Por otra parte, para los servidores se tiene un $95.41 \%$, un $4.53 \%$ menos que en el modelo principal de tres servidores y una cola. 


\section{Figura 16}

Porcentaje promedio de ocupación de los servidores en el sistema, modelo de 12 servidores y una cola.

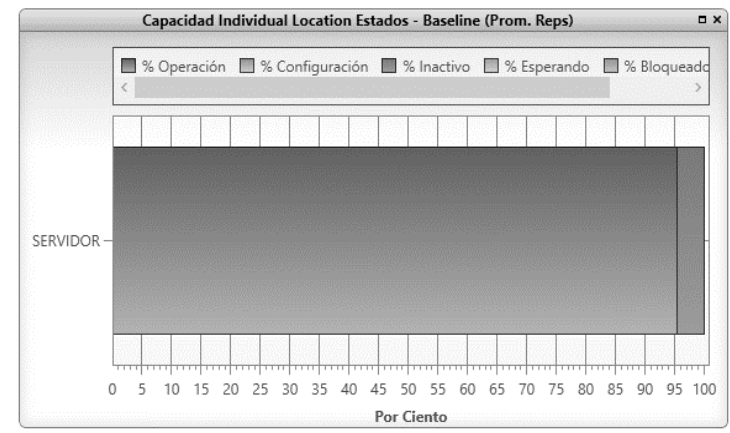

Nota. Fuente: Atencio, Bolaño y Díaz

Por otro lado, para la cola de espera se tiene un porcentaje de ocupación del $89.05 \%$, un $10.95 \%$ menos que el modelo principal de tres servidores y una cola.

Figura 17

Porcentaje promedio de ocupación de la línea de espera en el sistema, modelo de 12 servidores y una cola.

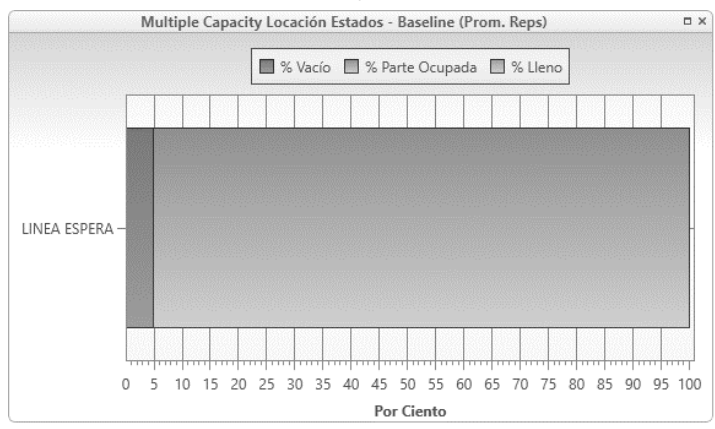

Nota. Fuente: Atencio, Bolaño y Díaz

De lo anterior se puede decir que, al añadir más servidores, el tiempo de espera de los clientes disminuye y, por tanto, la sobrecarga de los servidores también se aminora. A continuación, se muestran los resultados en una tabla con distintas configuraciones de servidores en contraste con el sistema original $\mathrm{M} / \mathrm{M} / 3$, evidenciando que el modelo actual no es el indicado para el sistema en producción. 
Tabla 7

Comparación de configuraciones de servidores del sistema.

\begin{tabular}{cccc}
\hline Modelo & $\begin{array}{c}\text { \% promedio de } \\
\text { espera del cliente }\end{array}$ & $\begin{array}{c}\text { \% promedio de } \\
\text { ocupación del servidor }\end{array}$ & $\begin{array}{c}\text { \% de ocupación } \\
\text { de la cola }\end{array}$ \\
\hline M/M/3 (actual) & 88,8 & 99,94 & 100 \\
M/M/15 & 70,12 & 95,41 & 89,05 \\
M/M/18 & 59,73 & 91,38 & 81,21 \\
M/M/20 & 50,07 & 85,81 & 71,42 \\
M/M/25 & 33,79 & 73,02 & 48,47 \\
\hline
\end{tabular}

Nota. Fuente: Atencio, Bolaño y Díaz

\section{Conclusiones}

Dentro del análisis expuesto es posible vislumbrar la factibilidad de agregar más servidores al sistema para mejorar el servicio, dado que con el modelo actual (M/M/3) el sistema no tiene la capacidad necesaria para atender largas líneas de espera puesto que se genera un cuello de botella en la atención al usuario. Los resultados de la experimentación demostraron que, al incrementar el número de servidores, los tiempos de espera por parte del usuario y el tiempo de atención disminuyen considerablemente, exponiendo que el sistema presenta una relación inversamente proporcional.

Los resultados tabulados en la tabla 7 muestran la disminución de la sobrecarga del sistema al balancear la carga entre los diferentes servidores, siendo el modelo $\mathrm{M} / \mathrm{M} / 15$ el que presenta mejoras significativas con el modelo $\mathrm{M} / \mathrm{M} / 3$.

Por lo anterior, es recomendable que el gerente a cargo de la sucursal modifique el sistema en busca de mejorar la calidad del servicio añadiendo 15 servidores, teniendo un total de 18.

Cabe mencionar que la simulación no es completamente infalible debido a que es la aplicación de un supuesto, no arregla el problema de fondo, solo ofrece una mejora temporal. 


\section{Referencias}

Jahn, B., Theurl, E., Siebert, U., \& Pfeiffer, K. (2010). Tutorial in Medical Decision Modeling Incorporating Waiting Lines and Queues Using Discrete Event Simulation. Value Heal, 13(4), 501-506. doi:https:// doi.org/10.1111/j.1524-4733.20

Abad, R. (2002). Introducción a la simulación ya la teoría de colas. Netbiblo.

Benites, J., \& Virhuez, K. (2019). Teoría de colas para la reducción del tiempo de ciclo de los ascensores de la torre principal de una universidad privada. Lima.

Cerutti, M., \& Valdaliso, J. (2003). Empresariado, industria y desarrollo regional en la periferia. Historia Mexicana. En Monterrey y Bilbao (1870-1914) (págs. 905-940).

De Arias, L., Montoya, L., \& Hen, S. (2010). Análisis de líneas de espera a través de teoría de colas y simulación. Sci. Tech, 3(46), 56-61.

Esfandiari, R., \& Dunna, M. (1996). Simulación y análisis de modelos estocásticos.

Gavilanes, S., Acosta, M., Gaibor, J., \& Tenorio, G. (2018). Cuellos de botella. Caribeña de Ciencias Sociales.

Gómez, F. (2011). Aplicación de teoría de colas en una entidad financiera: herramienta para el mejoramiento de los procesos de atención al cliente. Rev. Univ. EAFIT, 44(150), 51-63.

Márquez, I. (2010). La simulación como aprendizaje: educación y mundos virtuales. En Documento presentado en el II Congreso Internacional de Comunicación (Vol. 3).

Orrego, R. (2012). La Calidad del servicio, la gestión de flujos y la teoría de colas. Rev. Univ. EAFIT, 30(94), 23-29.

Pérez , J., \& Riaño, G. (2007). Análisis de colas para el diseño de una cafetería mediante simulación de eventos discretos. Rev. Ing, 12-21. 